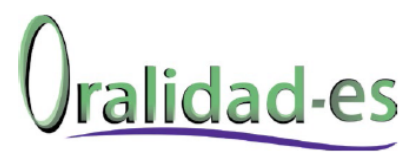

\title{
Un ambiente de aprendizaje para el desarrollo de procesos de lectura a través de la escucha en niveles iniciales de primaria
} A learning environment for development of reading processes through listening at first years of elementary

\author{
Danith Zorelly Castillo-Ruiz \\ danithcastillo@gmail.com \\ Universidad Distrital Francisco José de Caldas, \\ Colombia
}

Castillo-Ruiz, D. Z. (2020). Un ambiente de aprendizaje para el desarrollo de procesos de lectura a través de la escucha en niveles iniciales de primaria. Oralidad-es, 6, 1-19. https://revistaoralidad-es.com/index.php/ro-es/article/ view/118/128

Fecha de recepción: 04 de agosto de 2020 / Fecha de aceptación: 26 de diciembre de 2020 


\section{Resumen}

Este artículo presenta el resultado de una investigación cuyo objetivo fue la creación de un Ambiente de Aprendizaje (AA) que desarrollara procesos de lectura a través del audiolibro en niveles iniciales de primaria. Lo anterior a partir de la pregunta ¿Cuáles son los fundamentos pedagógicos y didácticos para un estudio didáctico orientado al diseño y posterior aplicación de una Trayectoria Hipotética de Aprendizaje (THA) que desarrolle procesos de comprensión lectora en español a través de la escucha? Considerando esto se realizó la fundamentación teórica, a partir de siete categorías vinculadas a la lectura, la didáctica, el audiolibro, las THA, el bilingüismo en español y los AA. Lo anterior permitió construir una THA que considerara los aprendizajes previos, los objetivos y las tareas que desarrollaran los procesos de lectura/escucha. Se concluye presentando la importancia de realizar un estudio didáctico previo a la enseñanza que impactará de manera significativa el aprendizaje.

\section{Palabras Clave}

Ambiente; aprendizaje; enseñanza; didáctica

\section{Abstract}

This article presents the result of an investigation that aimed to create a learning environment (LA) that will developed reading processes, through audiobook at initial levels of primary school. The aim was based on the question: What are the pedagogical and didactic foundations for a didactic study oriented to the design and subsequent application of a Hypothetical Learning Path that develops reading comprehension processes in Spanish, through listening? Thus, the theoretical foundation was made from seven categories related to reading, didactics, audiobook, Hypothetical Learning Trajectories, bilingualism in Spanish and LA. This allowed the construction of a HLP that considered previous learning, objectives and tasks that developed the reading / listening processes. Finally, the conclusions present the importance of conducting a didactic study prior to teaching that will significantly impact learning.

\section{Keywords}

Environment; learning; teaching; didactic 


\section{Introducción}

El interés por desarrollar un estudio didáctico que permita la creación de un Ambiente de Aprendizaje (AA) y el diseño de una Trayectoria Hipotética de Aprendizaje (THA) que tenga en cuenta lo saberes previos de los estudiantes surge a partir del análisis de un ambiente escolar que desarrolla su propuesta curricular desde el bilingüismo, comenzando con una inmersión en toda la etapa del preescolar en el idioma inglés hasta primero de primaria, grado en el que se abordaría el español como lengua materna desde una enseñanza formal de la lengua. Esta trayectoria propone la reflexión sobre el lugar del español en una población de niños y niñas que, por las condiciones académicas y sociales del contexto, se abordaría como segunda lengua.

Por lo anterior, el profesor partiría de la reflexión sobre las estrategias y condiciones didácticas que le permitirían al estudiante acceder al español. Es a partir de esta reflexión que surge la necesidad de realizar un estudio didáctico que dé paso a la creación de un ambiente de aprendizaje y al diseño de una THA que favorezca y potencie procesos de comprensión de lectura en español a través de la escucha. En relación con lo anterior, es importante puntualizar que el AA, según lo propuesto por Fernández (2015 citado en León et al., 2018), se entienden como

Un espacio en el cual se dan distintas interacciones entre los sujetos, es decir, estudiantes, docentes $y$ directivos, $y$ todos los componentes de un sistema de aprendizaje activo, razón por la cual los ambientes de aprendizaje se consideran como un espacio activo en el cual se mezclan los seres humanos, las acciones pedagógicas de quienes intervienen en la educación y un conjunto de saberes que son mediadores en la interacción de factores biológicos, físicos y psicosociales en un espacio que puede ser físico o virtual.
Es decir, que el AA no solo es el espacio físico, sino, también, el espacio de relaciones que se tejen entre todos los actores que se encuentran inmersos en la enseñanza y aprendizaje. Por consiguiente, se convierte en un concepto que permite realizar todo un estudio didáctico para, posteriormente, generar una THA que beneficie los procesos de lectura - escucha en niños de niveles iniciales, específicamente, del grado segundo del Colegio Gimnasio Vermont.

El propósito de realizar este estudio sobre el AA es aportar, desde la teoría del diseño, al posicionamiento de la didáctica como elemento principal en la formación del docente, ya que permite generar una reflexión de las prácticas educativas para, luego, impactar con aprendizajes significativos a los estudiantes, permitiendo visibilizar los procesos afectivos, cognitivos y sociales de la lectura. Esta última se entiende como una práctica viva que posibilita a los estudiantes avanzar en su conocimiento y reconocer su forma de ser y estar en el mundo.

Teniendo en cuenta lo anterior, esta propuesta está enfocada hacia la lectura escuchante, asumiendo que este tipo de lectura no ha sido muy desarrollada en la tradición teórica del campo del lenguaje ni en la didáctica del lenguaje. Por esta razón, se pretende valorarla como modalidad de lectura distinta a la del texto escrito, haciendo ampliación en la lectura como acción discursiva (Calderón et al., 2014). En ese sentido, se concibe leer de otras maneras y, cuando se tienen niños con una experiencia reducida en español (como es el caso de los estudiantes de grado segundo del Gimnasio Vermont), volver el aprendizaje de una nueva lengua objeto de un análisis particular, desde otros elementos que entran en juego como la lectura escuchante. Esto no solo permite la adquisición de la lengua, sino, también, la formación del sujeto escuchante.

Finalmente, es importante mencionar que el presente artículo se fundamenta en el trabajo de grado realizado por la autora en el Programa de 
Maestría en Pedagogía de la Lengua Materna de la Universidad Distrital Francisco José de Caldas (Bogotá, Colombia), culminado en el año 2020, el cual se tituló Una propuesta didáctica para el desarrollo de procesos de lectura-escucha en niveles iniciales de primaria.

\section{Referentes conceptuales}

Durante el desarrollo de la investigación se estudiaron aproximadamente 65 fuentes a partir de siete categorías fundamentales para generar una propuesta que lograra dar cuenta de un desarrollo de procesos de comprensión a través de la lectura-escucha. Sin embargo, se mencionan en este artículo conceptos generales que permitieron la construcción del AA y el diseño de una THA.

\section{La lectura como acción discursiva}

A lo largo del desarrollo en el campo del lenguaje han surgido diferentes estudios y cuestionamientos sobre el lugar que ocupa la lectura en el campo educativo. Así mismo, se han generado diversas aproximaciones teóricas y metodológicas frente a la inquietud de cómo llevarla a un escenario de enseñanza y aprendizaje. Para esta investigación es crucial comprender la lectura como acción discursiva, "básica para la interacción socio - cultural y para la actividad intelectiva" (Calderón, 2014, p. 117). Adicionalmente, desde una perspectiva sociocultural, en tanto se constituye como un camino para interactuar con otros. Lerner (2001) es clara cuando hace el llamado a la importancia de generar comunidades de lectores que encuentren en los textos diferentes perspectivas frente a sus cuestionamientos para así, poder construir una posición frente al mundo en el que habitan y también reconocer otras historias de vida, sentimientos y emociones que son validados a través de las discusiones que se tejen con los demás.
Desde esta misma perspectiva, Chartier (1993; 1999 citado por Rockwell, 2001) concibe la lectura como una práctica cultural que consiente que el diálogo entre lector y texto trasciende la decodificación y se alimente de nuevas comprensiones. Además, valida la importancia de la relación entre la lectura y la oralidad como una manera de expresarse y conectarse con los demás. De este modo, se posibilita asumir posturas críticas frente a las situaciones que se pueden desarrollar en el colegio, el barrio e incluso en la casa.

Otra perspectiva válida para el desarrollo de esta investigación, es la comprensión desde la estética de la lectura, que implica llevar al lector al plano de la imaginación, para que pueda inferir e identificar formas de ser de los personajes, lugares, ambientes, entre otros, ofreciendo una experiencia de lectura que permita la conexión e identificación con lo que se está leyendo. Por lo anterior, las palabras de Goodman (1996) cobran sentido al sostener que “(...) los niños que aprenden a disfrutar la lectura, llegado el momento de elegir, elegirán leer. Aquellos que aprenden a leer, pero no a disfrutar de la lectura, rara vez elegirán leer durante su tiempo libre” (p. 46). A su vez, Rosenblatt (1996) extiende la invitación a experimentarla como "un hecho único e individual que se percibe sólo en la mente y en las emociones de un lector en particular" (p. 1). Conviene subrayar que esta perspectiva propone al maestro pensar en textos que muevan la emocionalidad de los estudiantes y motiven el gusto por la lectura y proporcionen experiencias significativas desde la acción discursiva.

Esta perspectiva sociocultural de la lectura va más allá de los procesos de comprensión lectora abordados desde la psicolingüística, pero no los desconoce. Por el contrario, acude a la generación de hipótesis, la elaboración y confirmación de predicciones, entre otros procesos y operaciones en las que que intervienen los conocimientos del lector, los elementos contextuales y la situación comunicativa. De este modo, "lo que 
el lector ve en el texto y lo que él mismo aporta son dos subprocesos simultáneos y en estrecha interdependencia" (Colomer y Camps, 1996, p. 35). Por esto conviene articular los postulados de Solé (1992), orientados a las estrategias de lectura que puede realizar el profesor antes, durante y después de esta:

- Antes: ¿Qué tengo que leer?, ¿por qué/ para qué tengo que leerlo?, ¿qué sé yo acerca del contenido del texto?

- Durante: ¿Cuál es la información esencial que el texto proporciona y que es necesaria para lograr mi objetivo de lectura?, ¿tiene sentido este texto?, ¿se entiende lo que quiere expresar?, ¿qué dificultades plantea?

- Después: ¿Cuál es la idea fundamental que extraigo de aquí?, ¿puedo reconstruir las ideas contenidas en los principales apartados?, ¿tengo una comprensión adecuada de los mismos?, ¿cuál podrá ser el final de esta novela?, ¿qué sugeriría yo para solucionar el problema que aquí se plantea?, ¿qué le puede ocurrir a este personaje? etc. (Solé, 1992, p. 74)

Al concebir la lectura desde estas perspectivas se traza un camino claro frente a lo que se pretende alcanzar en términos de lectura con los estudiantes de primaria (grado segundo), apartándola de la decodificación y movilizándola a un plano social, cognitivo y crítico, en el que se aprende a leer para sentir, conmoverse, comprender, crear y elaborar discursos que se tejan con las opiniones de otros.

\section{La lectura a través de la escucha}

Teniendo en cuenta que la lectura es una práctica cultural que posibilita el diálogo entre lector, autor, texto y contexto que trasciende la decodificación y alimenta nuevas comprensiones, surge la posibilidad de concebir la lectura a través de la escucha como un fenómeno discursivo, necesario en la enseñanza del español y que no pretende ser mejor que la visual, pero que sí propone al maestro la posibilidad de adentrarse en ella y estudiar los procesos que emergen desde allí.

Según Kean y Personke (1976 citados por Beuchat, 1989), el niño ingresa a la etapa escolar con una escucha más desarrollada que el habla, pero al finalizar su paso por el colegio el habla adquiere más relevancia y la escucha se naturaliza, a tal punto, que se puede entender por qué es tan complejo para el individuo ponerse en el lugar del otro, respetar los turnos de la palabra y escuchar sin interrumpir. Esto sucede, según Beuchat (1989, p.1), porque "los libros guías o manuales, ofrecen muchas más estrategias para leer y escribir que para hablar, y mucho menos para escuchar". Al respecto, se observa que, generalmente, en el aula hay pocos espacios de diálogo y se limita a las actividades con los textos a una guía que responde a elementos únicamente gramaticales o de análisis de la obra, sin contemplar el sentir y las emociones vividas por el estudiante en torno al mismo.

Se considera que para desarrollar la escucha en el aula basta con poner a leer a los niños una parte específica del libro, pero estas acciones pocas veces pasan por el goce estético, quedándose únicamente en el proceso biológico, pues oír es diferente a escuchar, "oír se refiere a la recepción física de las ondas sonoras a través del oído (...) El escuchar puede definirse como el proceso por el cual el lenguaje hablado es convertido en significado en la mente" (Beuchat, 1989, p.2). Desde esta postura, vale la pena preguntarse ¿Qué de todo lo que ocurre en el aula de clase ha sido realmente escuchado por el niño? Esto dado que pasan casi nueve horas en el colegio y el currículo poco se cuestiona por trabajar esta acción discursiva. 
Reconociendo lo anterior, la intención de este trabajo investigativo es diseñar procesos que desarrollen la acción discursiva de leer a través de la lectura de audiolibros, buscando generar y analizar el vínculo entre escucha-lectura y los procesos que emergen a partir de esta relación que pueden ser interdependientes. Así, pues, se incluyen una serie de competencias, fases y procesos que permiten el fortalecimiento de esta acción discursiva. Por lo anterior, adquiere gran significación los niveles propuestos por Beuchat (1989):

- Escucha atencional: "el auditor focaliza su atención en un estímulo para obtener información y participar en forma activa” (p. 5).

- Escucha analítica: "se lleva a cabo cuando debemos analizar lo escuchado para responder o resolver algo" (p. 5).

- Escuchar apreciativo: "se realiza por goce, por el simple hecho de disfrutar y deleitarse con lo que se escucha” (p. 5).

En esta misma concepción de lectura escuchante, es imperativo contemplar las competencias postuladas por Lugarini (1995):

1. Competencia técnica: La capacidad de identificar y reconocer sonidos.

2. Competencia semántica: Relación propia entre significados y significantes.

3. Competencia sintáctica y textual: Identificar las relaciones sintagmáticas dentro del enunciado.

4. Competencia pragmática: Reconocer la intención y las características de la situación comunicativa en la que se da el mensaje.

5. Competencia selectiva: Se usa el mensaje con una finalidad.

Finalmente, articular las fases propuestas por Pavoni (citado en Lugarini, 1995):
* Pre-escucha: Se hace conciencia de por qué es importante escuchar y se crean expectativas en el estudiante para que pueda realizar anticipaciones.

* Escucha: Se mantiene la atención por medio de ejercicios y material de apoyo que estimulen los procesos de anticipación, verificación y relación.

* Pos-escucha: Se monitorea la comprensión y se integran actividades como pintar, escribir, actuar, debatir, etc.

De acuerdo con las anteriores competencias, fases y procesos, se propone comprender la lectura-escucha desde estos autores. Por consiguiente, se asume como práctica que da la apertura a estudiar, analizar y seguir paso a paso el proceso de aprendizaje que emerge al ponerlas en juego en un AA pensado para fortalecer su acción discursiva. Además, permite que el profesor comprenda que los procesos de comprensión de lectura visual y auditiva no difieren en gran medida y que se puede fortalecer a partir de estos dos sentidos.

\section{La didáctica del lenguaje}

Esta categoría se propone porque uno de los objetivos de esta investigación es hacer evidentes las relaciones de tipo didáctico que emergen en la construcción de un AA y el diseño una THA en lenguaje. Por tanto, la didáctica en esta propuesta se entiende como un

Campo teórico - práctico que articula explicaciones acerca de la naturaleza de las relaciones que se producen en el aula, es decir, las relaciones de enseñanza y aprendizaje, y por ello constituye también el campo natural de la acción del profesor (el que genera y orienta sus prácticas). En este sentido, el saber didáctico configura la idoneidad epistemológica del profesor y se caracteriza por ser un saber de tipo complejo, articulador de distintos elementos que, a la vez, permite 
generar articulaciones en el momento en que el profesor requiere concebir sus diseños didácticos, aplicarlos y evaluarlos. (Calderón et al., 2014, p. 101)

En ese sentido, es imperativo concebir la didáctica como la construcción y puesta en juego de las reflexiones del profesor, el estudio del contexto, la pertinencia de los contenidos y la búsqueda de estrategias para el desarrollo de la enseñanza y el aprendizaje significativos para la vida, pues es en la escuela principalmente donde al estudiante

Se le transmiten los modelos de vida, aprende a actuar como miembro de una "sociedad" -dentro y a través de los diversos grupos sociales, la familia, el vecindario, y así sucesivamente- $y$ a adoptar su "cultura", sus modos de pensar $y$ de actuar, sus creencias $y$ sus valores. (Halliday, 1979, p. 18)

$\mathrm{Al}$ tener el profesor conciencia de lo anterior se puede hablar de su idoneidad didáctica que se "caracteriza por ser un saber de tipo complejo, articulador de distintos elementos que, a la vez, permite generar articulaciones en el momento en que el profesor requiere concebir sus diseños didácticos, aplicarlos y evaluarlos" (Calderón et al, 2014, p. 101). Entonces, el docente debe instalar esta reflexión de manera fundamental en la puesta en escena de sus clases para que sea potenciada, investigada, escudriñada y, por lo tanto, significativa para sus estudiantes. El reto del maestro, entonces, es que la idea de

\section{Alfabetización permita resignificar (...) la lectura (...) a partir de una mirada más tematizadora hacia una más experiencial: el desarrollo de la lecturabilidad (...) como prácticas culturales que involucran múltiples contextos semióticos, estéticos y comunicati- vos $y$, por ello, multiplicidad de lectores (...) que de estas experiencias aprenden continua- mente (Calderón, et al., 2014, p. 74).}

Como consecuencia, es primordial que el docente reflexione sobre su práctica y cree situaciones de aprendizaje que permitan fortalecer la experiencia de la lectura.

\section{El audiolibro como dispositivo didáctico}

Al pensarse como dispositivo didáctico, se entiende el audiolibro como un Objeto de Aprendizaje (OA) que "está inmerso en el ambiente de aprendizaje y hace parte del diseño didáctico que lo estructura" (Calderón et al., 2016, p. 114). De modo que no se toma el audiolibro como un elemento que está aislado del diseño didáctico, sino que, más bien, este posibilita la modalidad de lectura a través de la escucha y, con ello, aprendizajes que se pretenden alcanzar.

El audiolibro propuesto para esta investigación fue la obra El superzorro de Roald Dahl, el cual se configura como un dispositivo didáctico a partir de la definición de los criterios para elegir la obra literaria en conjunto con los estudiantes, considerando que es una obra que brinda aportes a nivel social y estético. Así mismo, la puesta en escena de las reflexiones por parte del profesor respecto a la mediación del audiolibro en el proceso de aprendizaje se plantea considerando que

Quien escucha un audiolibro es estimulado a investigar sus contenidos y a adquirir posteriormente el texto impreso, sin excluir, al contrario, que el lector pueda agradecer escuchar los textos ya leídos en este nuevo formato que se está difundiendo como nueva oralidad en los últimos 20 años. (Vallonari, 2011, p. 47 citado en Castillo-Ruiz, Poveda y Vera, 2016, p. 48)

De manera que el dispositivo didáctico, en este caso el audiolibro, surge como un diseño que ha incluido reflexiones de tipo pedagógico, didáctico y tecnológico, y que se articula a unos aprendizajes situados que se espera puedan emerger en el momento de leer a través de la escucha. 


\section{Las Trayectorias Hipotéticas de Aprendizaje (THA)}

El término de Trayectoria Hipotética de Aprendizaje (THA) fue introducido por Simon (1995) $\mathrm{y}$ tiene como objetivo establecer predicciones frente a los aprendizajes que tienen los estudiantes, específicamente en el campo de las matemáticas. La apuesta que hace este trabajo de investigación es establecer una THA en el campo del lenguaje, considerando que

Una trayectoria hipotética de aprendizaje (THA) consiste en los objetivos para el aprendizaje de los estudiantes, las tareas matemáticas que se usarán para promover el aprendizaje de los estudiantes, y las hipótesis acerca del proceso de aprendizaje de los estudiantes (Simon citado por Gómez y Lupiáñez, s.f., p. 2).

De acuerdo con lo anterior, los objetivos permiten al profesor direccionar lo que se pretende alcanzar frente al aprendizaje y, luego, seleccionar las hipótesis y las tareas que funcionan de manera interdependiente, pues una beneficia el cumplimiento de la otra.

\section{Bilingüismo en español-inglés}

Esta categoría considera unos aspectos importantes a tener en cuenta para la enseñanza del español como segunda lengua, considerando el contexto en el que se desarrolla la investigación. Estos son:

- La autoestima y la confianza como base fundamental para las experiencias de aprendizaje.

- El conocimiento que el profesor debe tener de la lengua que está enseñando. En este caso, se considera la importancia de conocer los procesos que emergen a partir de la lectura - escucha en español como una posibilidad para aprender esta lengua.
- La importancia de determinar situaciones de comunicación concretas de uso de la lengua y, por ello, se propone una situación de oralidad a través de la escucha de textos literarios de tipo narrativo.

- El encuentro con el otro, proponiendo constantemente intercambios comunicativos en los que los niños se vean interesados en involucrarse en un diálogo, comunicando, en un primer momento, a través de la oralidad y, luego, a partir del dibujo y la escritura.

Por otro lado, tomar el enfoque interaccionista permite situar la obra, convirtiéndola en un objeto de discusión sociocultural y de reconocimiento de posturas respecto a diferentes elementos narratológicos que contiene, utilizar la lengua y escucharla, viviendo una situación auténtica de comunicación.

\section{Ambientes de Aprendizaje} (AA)

[T2] Ambientes de Aprendizaje (AA)

Es importante comprender el Ambiente de Aprendizaje (AA) como "un espacio en el cual se dan distintas interacciones entre los sujetos, es decir, estudiantes, docentes y directivos, y todos los componentes de un sistema de aprendizaje activo" (Fernández, 2015 citado en León, et al., 2018). Considerando que el AA es un espacio en el que emergen diferentes relaciones (estudiante - estudiante, estudiante - profesor, estudiante - aprendizaje, estudiante - enseñanza, entre otras), es importante para el profesor identificar fenómenos que pueden afectar el Ambiente y que no permiten que los objetivos que se proponen sean alcanzados, llevando al docente a tomar decisiones pedagógicas y didácticas. 
Al respecto, en el Módulo Cultiva del CADEP Acacia - UDFJC ${ }^{1}$, Calderón et al. (2019) propone el Curso de Ambientes de Aprendizaje Accesibles con Incorporación de Afectividad. En estos se ha considerado que los fenómenos que mayoritariamente afectan los AA son "la Deserción, el Bajo rendimiento académico, la Tensión didáctica y las Contingencias y cambios sociales educativos" (León y Bravo, 2018, p. 10). Específicamente para el AA que se pretende desarrollar en relación con los procesos de lectura - escucha en niños de segundo grado del Colegio Gimnasio Vermont se hacen evidentes dos fenómenos que es necesario definir y visibilizar. Estos son: el bajo rendimiento, entendido como "la no-conformidad, puntual o recurrente, de los resultados de un estudiante a un rango de evaluación académico específico (convencional o no convencional)" (León y Bravo, 2018, p. 10) y el fenómeno de la tensión didáctica, que se refiere a

las dificultades que van desde los problemas de comprensión o de relevancia del contenido por el estudiante (relación estudiante a contenido), pasando por la indiferencia del profesor por el contexto cognitivo o social del estudiante (profesor a estudiante), hasta la inadecuación del contenido a las posibilidades del profesor (contenido a profesor). (León y Bravo, 2018)

\footnotetext{
${ }^{1}$ El equipo Cultiva es el encargado de la construcción de una metodología para el diseño de Ambientes de Aprendizaje Accesibles y con Afectividad, en el marco del Proyecto ACACIA (Centro de Cooperación para el Fomento, Fortalecimiento y Transferencia de Buenas Prácticas que Apoyan, Cultivan, Adaptan, Comunican, Innovan y Acogen a la comunidad universitaria). Este proyecto fue financiado por el programa Erasmus + Capacity Building de la Unión Europea (2015-2018), realizado por un consorcio de 11 Universidades de América Latina y 3 de Europa. El propósito del proyecto fue fomentar la integración universitaria a partir del diseño y la validación de un sistema de Centros de Apoyo y Desarrollo Educativo Profesional - CADEP - en América Latina, mediante la cooperación intra e interinstitucional en la producción y desarrollo de recursos didácticos y tecnológicos, y en la generación de estrategias de fomento socio afectivo a poblaciones en riesgo de exclusión universitaria (Calderón et al., 2019). Para mayor información se puede acceder al sitio web del proyecto: https://acacia.red/blog/category/cultiva/
}

Para el AA de grado segundo del Colegio Gimnasio Vermont estos fenómenos parecen tener origen en la baja relación con el español que van desarrollando los estudiantes, ya que lo que se privilegia es la inmersión en inglés de los niños en el preescolar. Cuando inician el primer grado de primaria se encuentran ante el aprendizaje del español como lengua formal y, en algunos casos, para ellos no representa un tema de interés y/o consideran que no necesita estudio. Asimismo, las prácticas educativas del preescolar han propiciado un mayor estatus al inglés y, por ende, hay una creencia en los padres y los niños acerca de que toda la atención, el privilegio y el refuerzo deben enfocarse en el inglés y que el español se va adquiriendo a medida que pasa el tiempo.

\section{Metodología del diseño, estrategias didácticas y evaluación formativa}

Luego del estudio de cada una de las categorías propuestas a lo largo de este documento, se sitúa la metodología del diseño que, de acuerdo con Garello, Rinaudo \& Donolo (2011), tiene como objetivo "mejorar los programas de enseñanza y ampliar la base de conocimientos sobre los procesos de aprendizaje; es decir, que contribuye al desarrollo de teorías educativas" (p. 5). Para ello, en una primera etapa se realiza un estudio didáctico que permita dar respuesta a qué fundamentos epistemológicos, pedagógicos y discursivos se requieren para la formulación de una THA que favorezca el desarrollo de procesos de lectura en español como segunda lengua en niños de segundo grado.

A propósito de esto, se propone la lectura a través de la escucha por medio del audiolibro como dispositivo didáctico, entendiendo que las acciones discursivas de hablar y escuchar son experiencias primarias de los seres humanos y favorecen la aprehensión de una lengua, porque están dadas para actuar en lo familiar y lo cotidiano beneficiando las interacciones y 
al aprendizaje de la lengua en uso. De manera que la competencia de escucha debe ser puesta de forma intencionada, porque de ella emergen procesos, competencias, fases y etapas que permiten a los estudiantes afianzar su capacidad de escucha. Es por esto que, en este AA, se apuesta por una nueva e innovadora manera de leer, partiendo de la experiencia estética para, de manera progresiva, potenciar aspectos discursivos y gramaticales de la lengua, por medio de discusiones, piezas creativas, monólogos y creaciones de texto.

A continuación, y posterior al estudio didáctico realizado en esta investigación, se diseña una

Trayectoria Hipotética de Aprendizaje, que tiene por objetivo desarrollar procesos de lectura y escucha en un grupo de 24 niñas de grado segundo que estudian en el Colegio Gimnasio Vermont.

\section{Diseño de la THA para el desarrollo de procesos de lectura-escucha en niveles iniciales de primaria}

Esta propuesta didáctica se implementa a partir de la noción de Trayectoria Hipotética de Aprendizaje (THA) que consiste en tener en cuenta los siguientes aspectos: "los objetivos para el aprendizaje de los estudiantes, las tareas (...) que se usarán para promover el aprendizaje de los estudiantes, y las hipótesis acerca del proceso de aprendizaje de los estudiantes" (Simon, 1995 citado en Gómez y Lupiáñez, 2007, p. 2). Desde esa perspectiva, las actividades aquí propuestas comprenden tres partes: los objetivos, las tareas y las hipótesis que establecerán relaciones con la lectura a través de la escucha.

\section{Trayectoria Hipotética de Aprendizaje (THA)}

- Es posible favorecer la entrada a la actividad lectora de los niños de grado segundo a través de audiolibros.

- Contextos auténticos para la comprensión de textos en español, permitiendo el desarrollo de procesos de pensamiento relacionados con esta lengua.

- La experiencia de lectura a través de audiolibros puede mejorar procesos inferenciales para la comprensión de textos en español.

\section{Objetivos de aprendizaje}

- Ganar apreciación estética del estudiante frente a la lectura a través de la escucha.

- Desarrollar procesos de comprensión literal e inferencial a partir de la obra literaria $E l$ Superzorro del autor Roald Dahl.

- Integrar aprendizajes del aspecto gramatical de la lengua.

- Favorecer la construcción conjunta de valores.

El diseño de las actividades para la trayectoria sigue la estructura de Castillo, Poveda y Vera (2016) propuesto en el trabajo de pregrado titulado El audiolibro como elemento didáctico para el desarrollo de la lectura en poblaciones en contextos de diversidad: factor intelectivo. Esto debido a que en ese trabajo se alcanzó una estructura de unidad didáctica que compromete los distintos elementos que dan cuenta de las relaciones didácticas. En la tabla 1 se presenta el diseño de la situación didáctica. 
Tabla 1.

Diseño de la situación didáctica

\section{Situación Didáctica \\ Reconociendo las características de los personajes}

Proyecto: Una propuesta didáctica para el desarrollo de procesos de lectura-escucha en niveles iniciales de primaria

Previsión de recursos: el docente debe disponer de computador o grabadora con lector de CD y bafles (en el caso del computador).

Materiales presentes en el aula de clase: $1 / 8$ de cartulina para cada estudiante, marcadores, colores y diccionario.

Tópico: Comprensión lectora - nom- Audio seleccionado: "Los tres granjeros"

bres y cualidades

\begin{tabular}{|c|c|c|c|c|}
\hline $\begin{array}{l}\text { Actividad: ¿Y } \\
\text { quiénes son } \\
\text { Benito, Buñuelo y } \\
\text { Bufón? }\end{array}$ & Tiempo: & $\begin{array}{l}\text { Fase de pre-es- } \\
\text { cucha: } \\
10 \text { minutos }\end{array}$ & Fase de escucha: & $\begin{array}{l}\text { Fase posterior a la } \\
\text { escucha (conclusión - } \\
\text { evaluación): } \\
60 \text { minutos }\end{array}$ \\
\hline
\end{tabular}

\section{Objetivos de la actividad:}

- Desarrollar procesos de anticipación y verificación a partir del capítulo de la obra.

- Ganar apreciación estética con el estudiante.

- Identificar nombres y cualidades.

Propósitos/ hipótesis para el trabajo con el estudiante:

1. Se pretende que el estudiante

2. Reflexione acerca de las características de los personajes.

3. Pueda desarrollar procesos de comparación entre cualidades de personajes y las propias.

4. Cree un texto visual a partir de su comprensión y su experiencia del texto leído-escuchado.

Hipótesis para el trabajo con el estudiante: Si el estudiante identifica actitudes y acciones de los personajes podrá caracterizarlos.

\section{Rol del profesor:}

1. Preparación del escenario: Inicialmente adecúa el espacio en mesa redonda.

2. Desarrollo de la actividad: Orienta el desarrollo de la actividad y propone la valoración del proceso.

Rol del estudiante: Escucha los audios (en actitud de lectura), sigue las instrucciones, aporta los recursos solicitados, desarrolla las actividades y participa de manera activa en el proceso de evaluación.

\section{Desarrollo de la sesión:}

La actividad comprende una planeación en tres fases: pre-escucha, escucha y pos-escucha (conclusiones-evaluación).

Pre-escucha: Antes de iniciar con el proceso de lectura, se indaga con los niños ¿qué es la lectura para ellos?, ¿con qué partes del cuerpo podemos leer? Y ¿es posible leer escuchando? 
El profesor describe toda la actividad. Posteriormente, presenta la obra El superzorro y el capítulo que se va a leer/escuchar (se sugiere resaltar la importancia de la obra, el autor y el año de la obra), con el fin de ubicar en contexto al estudiante. El docente, luego de realizar esta presentación, siguiendo la sugerencia de Solé (1992) realiza las siguientes preguntas a los niños, con el objetivo de generar procesos de anticipación:

\begin{tabular}{|l|l|}
\hline \multicolumn{1}{|c|}{ Preguntas Solé (1992) } & $\begin{array}{c}\text { Transformación de la pregunta para los niños } \\
\text { de segundo }\end{array}$ \\
\hline ¿Qué tienen que leer? & ¿Qué vamos a leer?, ¿qué creen que es? \\
\hline ¿Por qué y para qué hay que leerlo? & ¿Por qué será importante leerlo? \\
\hline ¿Qué saben acerca del contenido del texto? & ¿Alguno sabe de qué se trata? \\
\hline ¿Con qué pueden relacionar el título de la obra? & ¿En qué piensan cuando leen El superzorro? \\
\hline
\end{tabular}

Fase de escucha: Los momentos de escucha analítica y apreciativa y de reconstrucción semántica se dan de manera simultánea. Sin embargo, se describen de manera individual para poder visibilizar los procesos que se esperan.

- Momento de escucha analítica y apreciativa: Los estudiantes escucharán/leerán el capítulo Los tres granjeros. El profesor diseña preguntas, siguiendo la propuesta Solé (1992), que los niños deben tener en cuenta en el momento de la lectura:

\begin{tabular}{|l|l|}
\hline \multicolumn{1}{|c|}{ Preguntas Solé (1992) } & \multicolumn{1}{|c|}{\begin{tabular}{c}
\multicolumn{1}{c|}{ Transformación de la pregunta para los niños } \\
de segundo
\end{tabular}} \\
\hline $\begin{array}{l}\text { ¿Cuál es la información importante que el texto } \\
\text { da y que es necesaria para lograr mi objetivo de } \\
\text { lectura? }\end{array}$ & $\begin{array}{l}\text { ¿Qué de lo que pasó para ti es lo más importan- } \\
\text { te? } \\
\text { ¿Quiénes participan? }\end{array}$ \\
\hline ¿Qué informaciones son poco relevantes? & $\begin{array}{l}\text { ¿De la historia que te cuentan qué es lo menos } \\
\text { importante? }\end{array}$ \\
\hline ¿Tiene sentido este texto? & $\begin{array}{l}\text { ¿Esta historia puede suceder aquí, en nuestra } \\
\text { ciudad, en nuestro país? }\end{array}$ \\
\hline ¿Se entiende lo que quiere expresar? & $\begin{array}{l}\text { ¿Has comprendido lo que ocurrió en la histo- } \\
\text { ria? }\end{array}$ \\
\hline ¿Qué dificultades plantea? & $\begin{array}{l}\text { ¿Qué dificultades se les presentan a los granje- } \\
\text { ros y el zorro? }\end{array}$ \\
\hline
\end{tabular}

- Momento de escucha: El docente, propone, a partir del texto escuchado (reconstrucción semántica), que cada estudiante divida la cartulina en tres partes y, a medida que va escuchando/leyendo el capítulo, va dibujando cada uno de los granjeros. La intención será que los niños puedan realizar anticipaciones, es por esto que en determinados momentos se pausará al audiolibro, para que cada niño pueda dibujar lo que imagina.

Posteriormente, escucharán/leerán todo el capítulo y podrán verificar si lo que dibujaron corresponde o no al personaje que imaginaron y realizarán el dibujo de este con las características indicadas en el audiolibro. 
- Momento de escritura: Se pedirá a los niños que, debajo del dibujo, escriban ¿cómo se llaman los personajes?, ¿cómo son físicamente? y ¿cómo es su personalidad?

En este momento se incluirá el recurso del diccionario para hacer consultas de las palabras desconocidas o que tengan duda frente a cómo se escriben (previamente se les ha enseñado a usarlo, en el grado primero).

\section{Fase pos-escucha (conclusiones-evaluación):}

- Momento de valoración de la actividad y conceptualización de los términos nombre-sustantivo y cualidad-adjetivo: Se realizarán, en mesa redonda, las siguientes actividades:

a. Se divide a los estudiantes en tres grupos, cada uno tendrá un granjero de los tres y se encargará de seleccionar un dibujo y enriquecerlo con palabras para, luego, socializarlo a sus compañeros con las características atribuidas. El profesor se encargará de ir tomando nota de estas en el tablero.

b. Posteriormente, se pregunta a los estudiantes si alguno sabe qué es un sustantivo y un adjetivo. Se escribirán las ideas de los niños y, finalmente, la docente realiza la conceptualización a partir de la actividad de la obra.

- Momento de creación de texto escrito: ¿Quién y cómo soy yo? A partir de toda la actividad se propone a los estudiantes pintarse en $1 / 4$ de cartulina y escribir el sustantivo (nombre) y los adjetivos (características físicas y de su personalidad). Esta actividad se realizará en casa y será expuesta en las siguientes clases como una oportunidad para reconocerse entre ellos mismos. Cada estudiante deberá escribir mínimo tres cualidades positivas que destaquen sus habilidades.

\section{Evaluación de la actividad:}

La evaluación de esta actividad se propone en los niveles estético e intelectivo propuestos por Castillo, Poveda y Vera (2016) . Desde el nivel estético se pretende observar qué grado de apreciación y valoración de los textos desarrolla el niño a partir de la lectura/escucha del capítulo de la obra, dejando comprender al profesor, a través de sus actitudes, si cumple y en qué grado con el objetivo estético de la actividad y si logró que el estudiante tuviera una escucha apreciativa.

Complementariamente, desde el nivel intelectivo la evaluación de esta actividad permite observar el desarrollo de anticipaciones y uso de cualidades-adjetivos y nombres-sustantivos.

\footnotetext{
${ }^{2}$ Fueron construidos a partir del trabajo de pregrado titulado: El audiolibro como elemento didáctico para el desarrollo de la lectura en poblaciones en contextos de diversidad: factor intelectivo, en el marco del proyecto Desarrollo didáctico y tecnológico en escenarios didácticos para la formación de profesores que acogen la diversidad: factores para su implementación y su validación en la UDFJC (código Colciencias 1419-6614-44765-4), el cual se puede consultar en: http://repository.udistrital.edu.co/bitstream/11349/3899/1/ CastilloRuizDanithZorelly2016.pdf
} 


\begin{tabular}{|c|c|c|}
\hline \multicolumn{3}{|c|}{ NIVEL ESTÉTICO } \\
\hline Proceso / Actitud & Indicador del proceso & $\begin{array}{l}\text { Valoración } \\
\text { cualitativa } \\
\end{array}$ \\
\hline $\begin{array}{l}\text { Interés } \\
\text { Según el DRAE, una acep- } \\
\text { ción de interés es: "m. In- } \\
\text { clinación del ánimo hacia } \\
\text { un objeto, una persona, } \\
\text { una narración, etc." }\end{array}$ & $\begin{array}{l}\text { - El estudiante muestra una actitud de lectura } \\
\text { a través de la escucha. } \\
\text { El estudiante sigue las instrucciones del } \\
\text { profesor y asume la actitud de leer /escuchar } \\
\text { y prestar atención a los detalles que trae la } \\
\text { narración del capítulo. } \\
\text { - El estudiante aporta características de los } \\
\text { personajes. } \\
\text { - El estudiante se integra y muestra entusias- } \\
\text { mo por las actividades realizadas. } \\
\text { El estudiante expresa interés por lo que } \\
\text { dicen su profesor y sus compañeros. } \\
\text { El estudiante muestra interés por el signifi- } \\
\text { cado de los nombres-sustantivos y cualida- } \\
\text { des-adjetivos. }\end{array}$ & $\begin{array}{l}\text { Sugerencia: consig- } \\
\text { nar datos observados } \\
\text { durante el proceso } \\
\text { que permitan valorar } \\
\text { la relación proceso- } \\
\text { indicador }\end{array}$ \\
\hline $\begin{array}{l}\text { Con templación } \\
\text { Según el DRAE, una acep- } \\
\text { ción de contemplación } \\
\text { es: “-f. Consideración, } \\
\text { atención o miramiento } \\
\text { que se guarda a alguien. I o } \\
\text { a alguna cosa”. }\end{array}$ & $\begin{array}{l}\text { - El estudiante expresa sorpresa, interés o ale- } \\
\text { gría mediante la expresión facial y mantiene } \\
\text { la atención durante toda la lectura/escucha. } \\
\text { - Expresa alegría, acuerdo o desacuerdo por } \\
\text { las actividades que se realizan. } \\
\text { - El estudiante tiene actitud de cuidado y } \\
\text { respeto por los otros y por los materiales que } \\
\text { se emplean. } \\
\text { El estudiante manifiesta empatía por el ca- } \\
\text { pítulo escuchado - leído y por los aportes de } \\
\text { sus compañeros. }\end{array}$ & \\
\hline $\begin{array}{l}\text { Actitud ética } \\
\text { Se manifiesta y se valora } \\
\text { una actitud de observación } \\
\text { de las reglas de conviven- } \\
\text { cia. }\end{array}$ & $\begin{array}{l}\text { - El estudiante observa y respeta los turnos de } \\
\text { - } \text { la palabra. } \\
\text { riales y los recursos del entorno. } \\
\text { - El estudiante trata con cuidado a sus compa- } \\
\text { ñeros. } \\
\text { - El estudiante reconoce actitudes que afectan } \\
\text { la convivencia. } \\
\text { El estudiante manifiesta actitud de trabajo en } \\
\text { equipo aportando ideas y escuchando las de } \\
\text { los otros. }\end{array}$ & \\
\hline
\end{tabular}




\begin{tabular}{|c|c|c|}
\hline \multicolumn{3}{|c|}{ NIVEL INTELECTIVO } \\
\hline Proceso / Actitud & Indicador del proceso & $\begin{array}{l}\text { Valoración } \\
\text { cualitativa }\end{array}$ \\
\hline $\begin{array}{l}\text { Inferenciales } \\
\text { Según el DRAE, una } \\
\text { acepción de inferencia es: } \\
\text { "tr. Deducir algo o sacarlo } \\
\text { como conclusión de otra } \\
\text { cosa". }\end{array}$ & $\begin{array}{l}\text { - El estudiante realiza anticipaciones a partir } \\
\text { de la escucha/lectura del capítulo. } \\
\text { El estudiante propone características (adje- } \\
\text { tivos) a los personajes a partir de la escucha/ } \\
\text { lectura. } \\
\text { - El estudiante identifica nombres-sustantivos } \\
\text { en los personajes del capítulo. }\end{array}$ & $\begin{array}{l}\text { Sugerencia: Consig- } \\
\text { nar datos observados } \\
\text { durante el proceso } \\
\text { que permitan valorar } \\
\text { la relación proceso- } \\
\text { indicador }\end{array}$ \\
\hline $\begin{array}{l}\text { Creatividad } \\
\text { Según el DRAE, una } \\
\text { acepción de crear es: "tr. } \\
\text { Establecer, fundar, in- } \\
\text { troducir por vez primera } \\
\text { algo; hacerlo nacer o darle } \\
\text { vida, en sentido figurado". }\end{array}$ & $\begin{array}{l}\text { - El estudiante crea el dibujo de los personajes } \\
\text { a partir de la escucha/lectura. } \\
\text { El estudiante propone nuevos significados y } \\
\text { nuevas situaciones. } \\
\text { - El estudiante construye a partir de sí mismo } \\
\text { su imagen y hace uso de las cualidades-ad- } \\
\text { jetivos y nombres-sustantivos para contar } \\
\text { quién es y cómo es. }\end{array}$ & \\
\hline $\begin{array}{l}\text { Uso } \\
\text { Según el DRAE, una } \\
\text { acepción de uso es: "m. } \\
\text { Capacidad o posibilidad de } \\
\text { usar algo". }\end{array}$ & 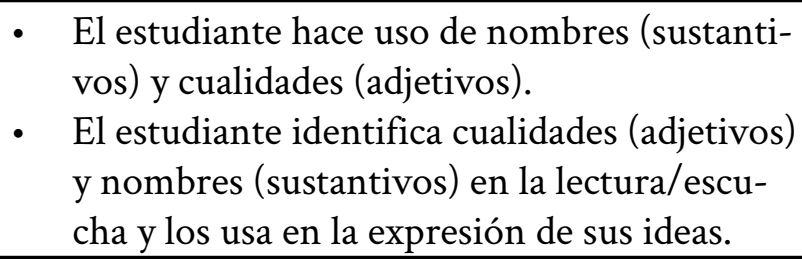 & \\
\hline
\end{tabular}

Fuente: adaptada de la estructura de Castillo-Ruiz, Poveda y Vera

(2016) propuesta en el trabajo de pregrado El audiolibro como

elemento didáctico para el desarrollo de la lectura en poblaciones en

contextos de diversidad: factor intelectivo. 


\section{Análisis y discusión de resultados}

A partir de la aplicación de la situación didáctica Reconociendo las características de los personajes en grado segundo, con 25 niñas del Colegio Gimnasio Vermont se visibilizaron los alcances de las actividades propuestas en relación con los objetivos que sostienen la THA. El posterior análisis y discusión de los resultados se plantea en relación con esta THA.

Hipótesis 1: Es posible favorecer la entrada a la actividad lectora de los niños de grado segundo a través de audiolibros. En efecto se evidencia un goce estético de la obra que se visibiliza a través de los gestos, los dibujos, las palabras y la actitud comprensiva frente al texto, que se revela en su acuerdo con lo que construyen otras niñas y ellas mismas. Por lo tanto, se plantea que efectivamente hay un proceso de lectura.

\section{Hipótesis 2: Contextos auténticos para la comprensión de textos en español permitirá el desarrollo de procesos de pensamiento relacionados con esta lengua. $\mathrm{Al}$ permitirles} hablar desde lo que ellas saben y les interesa ampliaron su vocabulario y se cuestionaron frente a cómo escribir palabras que pudieran completar las descripciones de los granjeros y de ellas mismas. Por otro lado, hay una etapa en relación con la lectura del capítulo y dar razón de este, preguntar sobre las palabras, el significado, la ortografía y luego se da la etapa de creación, a partir de un contexto auténtico que busca que las niñas indaguen y reconozcan sus propias características y, posteriormente, entre ellas mismas las reconfirmen.

Hipótesis 3: La experiencia de lectura a través de audiolibros puede mejorar procesos inferenciales para la comprensión de textos en español. El haber hecho la lectura-escucha del capítulo de Los granjeros les permitió hacer inferencias y conexiones con temas de la vida diaria, los campesinos, el maltrato animal, entre otros. Así mismo, hubo una relación entre las características escuchadas y lo que dibujaban y una inferencia frente a cómo se representaban físicamente y cómo tenían similitudes con los campesinos de nuestro país.

Finalmente, lo aprendido lo traían a su propia representación, haciedo la diferencia entre lo físico y lo emocional (mis características corresponden a dos categorías diferentes). Al respecto, se destacan manifestaciones de la aprehensión de la lengua a través de la escritura.

\section{Conclusiones}

El estudio didáctico realizado en esta investigación permitió poner en escena situaciones didácticas cercanas y acordes a la realidad de una población de estudiantes de segundo de primaria. Puntualmente, la construcción de la THA puso en consideración muchos factores del ambiente que, en principio, no se contemplaban, pero que cobraron valor en la medida en la que se observaron y examinaron de manera detallada en los procesos que se propusieron. Así, pues, volver sobre el contexto lingüístico que permea la vida de los niños fue clave. También lo fue considerar las relaciones de los estudiantes con el español y contemplar su enseñanza como segunda lengua, lo cual permitió afinar y fortalecer las actividades propuestas para realmente impactar el aula de clase.

Del mismo modo, identificar la relación de las niñas con la actividad lectora permitió comprender muchas de las dinámicas que tenían hacia la asignatura y, desde esa identificación, generar ambientes de lectura que, en principio, buscaron el goce estético: la sorpresa, el asombro y la risa como parte de la entrada a un mundo que es desconocido, pero que estaba listo para recibirlas. Este tipo de experiencia fue posible al proponer la escucha como modalidad de lectura y, desde esa perspectiva, identificar los procesos implicados y observarlos con detenimiento intentando 
no perder ningún detalle, afirmando la premisa de que la lectura a través de la escucha es posible y que es viable en tanto los niños se sensibilizan y toman de este tipo de lectura elementos para hacer relaciones con su realidad y, por consiguiente, proponer diferentes tipos de inferencias que son validadas y acordes a los procesos esperados.

No obstante, esto fue posible debido al diseño de las tareas, que tenían por objetivo favorecer el acto lector y por esto fueron pensadas en un contexto específico y bajo unas condiciones propias que buscaron y lograron la sensibilización de las niñas, el reconocimiento de sí mismas y de la voz del otro. Además, desarrollaron una postura propia frente a la aprobación o desaprobación de las actitudes de los personajes y privilegiaron la interacción como una herramienta valiosa, que permite la construcción de aprendizajes colectivos que traspasan la evaluación numérica.

Por otro lado, favorecer la accesibilidad y la afectividad permitió configurar diseños didácticos que realmente acogieron a todos. Es por esto que se debe pensar en la accesibilidad no solo para personas ciegas, sordas o con algún tipo de discapacidad, sino como un atributo que debe ser necesario tener en cuenta en el diseño didáctico que genera el profesor, pues el contexto y los estudiantes son diversos, pero el aprendizaje debe posibilitarse a todos.

Así mismo, las THA se proponen como una nueva estrategia que debe ser estudiada y explotada en los diferentes contextos, si bien esta es una propuesta, la invitación queda abierta a todos los docentes de diferentes áreas del conocimiento a hacer sus propios diseños.

Finalmente, este proceso en la formación como docente investigadora fue enriquecedor en tanto constantemente y a lo largo de estos dos años de investigación me llevó a reflexionar sobre lo pensado, lo aplicado y lo escrito, y a cuestionar mi quehacer diario que, en ocasiones, puede convertirse en rutinario y, debido a esto, ir perdiendo la esencia de la innovación y del papel social de la enseñanza de la lengua en la educación. Ser docente investigador implica ir más allá de un horario laboral establecido, y buscar incansablemente teoría que nutra las concepciones que uno ha ido formando en su ser. Además, reflexionar y no dejar los planteamientos como verdades inamovibles, sino como aquello que tiene que cambiar, pues no todos los niños son iguales y es un deber ser como docente propiciar cambios que beneficien el aprendizaje y la enseñanza.

En ese sentido, la metodología del diseño aportó considerablemente a mis reflexiones en el proceso, pues realizar un estudio didáctico es imperativo cuando se quiere investigar en educación y hacerlo fue el paso primordial para que la propuesta de THA favoreciera, realmente, lo que se estaba buscando y, así, evitar caer en divagaciones e imprecisiones que afectarían a las niñas y, luego del camino de reflexión y construcción, la satisfacción de generar un aporte en el campo de la lengua, plantear otras maneras de concebir la enseñanza del español e invitar a explorar la lectura desde otros sentidos. 


\section{Referencias}

Beuchat C. (1989). Escuchar: el punto de partida. Lectura y vida. Revista Latinoamericana de Lectura, (3), 20-25. http://www.lecturayvida.fahce. unlp.edu.ar/numeros/a10n3/10_03_Beuchat. pdf

Bravo, F y León, O (2017). Metodología de diseño de ambientes de aprendizaje accesibles con incorporación de afectividad. ACACIA Cultiva.

Calderón, D. (Ed.). (2014). Referentes curriculares con incorporación de tecnologías para la formación del profesorado de lenguaje y comunicación en y para la diversidad. Universidad Distrital Francisco José de Caldas.

Castiblanco, R y León, O (2018). Referente accesibilidad para ambientes de aprendizaje. ACACIA Cultiva.

Castillo, D. (2016). El audiolibro como elemento didáctico para el desarrollo de la lectura en poblaciones en contextos de diversidad: factor intelectivo, en el marco del proyecto "desarrollo didáctico y tecnológico en escenarios didácticos para la formación de profesores que acogen la diversidad: factores para su implementación y su validación en la UDFJC (código Colciencias: 14196614-44765-4) [Tesis de pregrado, Universidad Distrital Francisco José de Caldas]. https:// repository.udistrital.edu.co/bitstream/ handle/11349/3899/CastilloRuizDanithZorelly2016.pdf;jsessionid=E9C7A6D699F1DE0D3D2B041CD957 A927? sequence $=1$

Castillo, D. (2020). Una propuesta didáctica para el desarrollo de procesos de lectura-escucha en niveles iniciales de primaria [Tesis de maestría, Universidad Distrital Francisco José de Caldas]. Repositorio Institucional Universidad Distrital Francisco José de Caldas. https://repository.udistrital.edu.co/hand$\underline{\text { le/ } 11349 / 24396}$
Clements, D. H. \& Sarama, J. (2004). Learning trajectories in mathematics education. Mathematical thinking and learning, 6(2), 81-89. https://www.tandfonline.com/doi/ abs/10.1207/s15327833mt10602_1

Colomer, T., y Camps, A. (1996). Enseñar a leer, es enseñar a comprender. Celeste Ediciones.

Garello, M. V., Rinaudo, M. C. \& Donolo, D. (2011). Valoración de los estudios de diseño como metodología innovadora en una investigación acerca de la construcción del conocimiento en la universidad. Revista de Educación a Distancia, 5, 1-34. http://www.um.es/ead/ reddusc/5

Gómez, P y Lupiáñez, J. (2007). Trayectorias Hipotéticas de Aprendizaje en la Formación Inicial de Profesores de Matemáticas de Secundaria. PNA: Revista de investigación en Didáctica de la Matemática, (1), 1-22. https://dialnet. unirioja.es/descarga/articulo/2238351.pdf

Goodman, K. (1996). La lectura, la escritura y los textos escritos: una perspectiva transaccional sociopsicolingüística. Textos en contexto. $N^{\circ} 2$ : Los procesos de lectura y escritura (pp. 5-68). Asociación Internacional de Lectura.

Halliday, M. (1979). El lenguaje como semiótica social. La interpretación del lenguaje y el significado. Fondo de Cultura Económica.

Lerner, D. (2001). Leer y escribir en la escuela: lo real, lo posible y lo necesario. Fondo de Cultura Económica.

Lugarini, E. (1995). HABLAR Y ESCUCHAR. Por una didáctica del "saber hablar" y del "saber escuchar". Signos. Teoría y práctica de la educación, 30-51. http://www.quadernsdigitals.net/datos/hemeroteca/r_3/ nr_42/a_638/638.html 
Poveda, D. \& Vera, D. (2016). El audiolibro como elemento didáctico para el desarrollo de la lectura en poblaciones en contextos de diversidad: factores epistemológico y comunicativo, en el marco del proyecto "desarrollo didáctico y tecnológico en escenarios didácticos para la formación de profesores que acogen la diversidad:factores para su implementación y su validación en la UDFJC (código Colciencias: 1419-6614-44765-4) [Tesis de pregrado, Universidad Distrital Francisco José de Caldas]. https://1library. co/document/yr3r318y-audiolibro-didactico-desarrollo-poblaciones-contextos-diversidad-epistemologico-comunicativo.html

Rockwell, E. (2001). La lectura como práctica cultural: conceptos para el estudio de los libros escolares. Educação e Pesquisa, (27), 11-26. https://www.scielo.br/scielo.php?pi$\underline{\mathrm{d}=S 1517-7022001000100002 \& \text { script }=\text { sci_abs- }}$ tract\&tlng=es

Solé, I. (1992). Estrategias de lectura. Editorial Graó. 